\title{
Perfil epidemiológico de casos HIV/Aids cadastrados em Serviço Ambulatorial Especializado
}

Epidemiological profile cases HIV/Aids registered in Specialized Outpatient Service Perfil epidemiológico del VIH/Sida registrados en Servicios de Consulta Especializada

Nádya Thalita Novaes dos Santos1, Susanne Pinheiro Costa e Silva2, Flávia Emília Cavalcante Valença Fernandes 3, Leilane Dias

Santana 4, Tuanny Italla Marques da Silva ${ }^{5}$

\section{Resumo}

Esta pesquisa objetivou conhecer o perfil epidemiológico dos usuários atendidos no serviço de referência para IST/HIV/Aids em Petrolina-PE. Trata-se de estudo descritivo com abordagem quantitativa, no qual foram analisadas fichas de notificação e investigação de HIV/Aids entre 2010 e 2014, totalizando 331 notificações. Observou-se evolução no número de notificações de casos Aids no período de 2009 a 2014, sendo a maioria do sexo masculino $(58,3 \%)$, com faixa etária predominante de 31 a 50 anos (52,9\%). Mais de $50 \%$ dos casos envolvia pessoas com baixa escolaridade. O provável modo de transmissão prevalente era por via sexual, mesmo nos casos com nível elevado de escolaridade, sendo os heterossexuais a categoria de maior exposição. Os sinais e

\footnotetext{
1 Residente em Urgência e Emergência. Enfermeira. Universidade Federal do Vale do São Francisco. PetrolinaPE, Brasil.

${ }^{2}$ Departamento de Enfermagem em Saúde Coletiva. Docente do Mestrado Profissional em Gerontologia.

3 Doutoranda em Inovação Terapêutica. Docente da Universidade de Pernambuco/UPE.

${ }^{4}$ Enfermeira.

${ }^{5}$ Residente em Saúde Coletiva. Enfermeira. Universidade Pernambuco/UPE.
}

sintomas de definição de caso prevalentes foram astenia, caquexia, diarreia crônica, febre persistente e contagem de Linfócitos CD4+ menor do que 350 cél $/ \mathrm{mm}^{3}$. O perfil traçado demonstrou aumento de casos no sexo feminino, embora a maioria ainda seja do sexo masculino com baixo nível de instrução. Mesmo assim, a ausência de dados configurou-se como fator limitante para análise mais detalhada, impedindo que outros elementos pudessem ser identificados. Conhecer tais achados pode auxiliar à formulação de ações específicas que promovam educação e diminuam a cadeia de transmissão.

Descritores: Perfil de saúde; HIV; AIDS; Pacientes.

\begin{abstract}
This study aimed to know the epidemiological profile of the users serviced by the referral service for HIV/AIDS in the city of Petrolina, State of Pernanbuco. It is a descriptive study with a quantitative approach in which 331 HIV/AIDS notification and investigation files were analyzed between 2010 and 2014. The number of AIDS cases' notifications within
\end{abstract}


2009 and 2014 was observed, revealing a majority of males (58.3\%) aged between 31 and 50 years $(52.9 \%)$. Also, more than $50 \%$ of the cases were composed of low schooling people. Sex was accounted as the probable prevailing way of transmission, even in cases of a high educational level, being the heterosexuals the most exposed category. The prevailing signs and symptoms of case definition were asthenia, cachexia, chronic diarrhea, persistent fever, and CD4 + lymphocyte count $<350$ cells / mm3. The research showed an increase in female cases, although the majority is still composed of low schooling male. The lack of data was a limiting factor for a more detailed analysis, preventing other elements from being identified. The knowledge of such findings can help formulate specific actions so to promote education and reduce the transmission chain.

Key words: Health Profile; HIV; Aids; Patients.

\section{Resumen}

El estudio objetivo conocer el perfil epidemiológico de los pacientes atendidos en el servicio de referencia para VIH/SIDA en Petrolina-PE. Estudio descriptivo con enfoque cuantitativo en el que se analizaron las formas de presentación de informes y la investigación del VIH/SIDA entre 2010 y 2014, un total de 331 notificaciones. Aumento en el número de notificaciones de casos de SIDA desde 2009 hasta 2014, la mayoría de los varones (58.3\%) y de edades entre 31 y 50 años (52,9\%). Más del $50 \%$ de los casos fueron personas con bajo nivel de educación. El modo predominante de transmisión probable era a través de las relaciones sexuales; los heterosexuales y la categoría de mayor exposición. Los signos y síntomas de definir el caso más frecuente fueron astenia, caquexia, diarrea crónica, fiebre y persistente de linfocitos CD4+ inferior a 350 células/mm3. El perfil mostró un aumento de casos entre las mujeres, pero aún así sigue siendo en su mayoría hombres, con bajos niveles de educación. Sin embargo, la ausencia de datos se ha configurado como un factor limitante para su posterior análisis. El conocimiento de estos hallazgos puede ayudar a formular acciones para promover la educación y reducir la cadena de transmisión.

Descriptores: Perfil de salud; VIH; Sida; Pacientes.

\section{Introdução}

Decorridas mais de três décadas após o surgimento do vírus da imunodeficiência humana (HIV), a síndrome da imunodeficiência adquirida (Aids) ainda é considerada uma pandemia e um grave problema de saúde pública também no Brasil, mesmo que estudos demonstrem possível 
tendência de estabilização em toda a América Latina. Os continentes Africano e Asiático são os líderes nas taxas de incidência e prevalência do vírus, ficando a América Latina na terceira posição ${ }^{(1)}$.

O Brasil tem registrado anualmente cerca de 41 mil casos de Aids nos últimos cinco anos. Desde a identificação do primeiro episódio em 1982 até junho de 2016, foram registrados mais de 800 mil casos da doença, com número superior a 300 mil mortes até 2015 no país ${ }^{(2)}$.

Na década de 1980, a Aids atingiu principalmente homossexuais do sexo masculino, profissionais do sexo, usuários de drogas injetáveis e portadores de hemofilia, todos eles considerados grupos de risco para contaminação. Atualmente, a exposição heterossexual entre homens e mulheres tem aumentado vertiginosamente. As principais fontes de transmissão do vírus HIV são o sangue, secreção vaginal, sêmen e leite materno, sendo a via sexual a mais comum no país ${ }^{(3)}$.

Com isso, a infecção por HIV/Aids vem sofrendo um processo de mudança no perfil epidemiológico, demonstrada pela intensificação da heterossexualização, feminização e juvenização, o que contribuiu para melhor percepção sobre a magnitude da doença e noção de vulnerabilidade a ela. Atualmente, não se admite mais a classificação por grupos de risco ou até mesmo comportamentos de risco para contrair
HIV/Aids, mas, sim, o grau de vulnerabilidade em que está exposto o indivíduo ${ }^{(3-4)}$.

Em 2015, as adolescentes e as jovens com idades de 15 a 24 anos foram responsáveis por $20 \%$ das novas infecções por HIV em todo o mundo, havendo certo desequilíbrio de gênero e disparidades de acordo com a localidade, o que demonstra a necessidade de enfrentar a infecção de múltiplas maneiras no intuito de alcançar os índices estimados para a exclusão da AIDS como ameaça à saúde pública até 2030 . Estima-se que em 2015 existiam no mundo 36,7 milhões de pessoas infectadas pelo HIV, entre homens, mulheres e crianças. Apenas naquele ano, 2,1 milhões de pessoas foram infectadas pelo vírus. Mesmo assim, várias entidades comemoram a estabilização e até mesmo a redução do número de novas infecções em muitos países ao redor do mundo, incluindo localidades da África e Ásia (5).

O panorama brasileiro segue, em grande parte, o que é observado em nível mundial. Nas regiões Centro-oeste, Sul e Sudeste houve estabilização ou queda da taxa de detecção de Aids nos últimos anos. Em contrapartida, quando observada isoladamente, a região Nordeste do Brasil apresenta tendência linear de crescimento dessa taxa; em 2006, registraram-se 11,2 casos por 100 mil hab., enquanto em 2015 esse índice cresceu para 15,3, representando 
aumento de $37,2 \%$, o que também ocorreu com a região Norte, em proporções ainda maiores ${ }^{(2)}$.

Face ao desafio de prevenir e controlar a epidemia, autoridades governamentais e sociedade civil articulam-se para expandir o conhecimento e o emprego de tecnologias que possam aperfeiçoar a qualidade de vida, investindo nas medidas de prevenção e de atenção àqueles que convivem com HIV/Aids, mesmo sem uma possível cura ainda estabelecida ${ }^{(5-6)}$.

As políticas públicas brasileiras de prevenção e controle do HIV/Aids ganharam destaque pelas diferentes entidades internacionais como referência nas ações realizadas em âmbito nacional e pela colaboração com outros países em desenvolvimento. Suas estratégias estão fixadas em três pilares principais de intervenção, sendo eles: vigilância epidemiológica, prevenção e assistência ${ }^{(3)}$.

Uma das principais ferramentas para tal feito foi a implantação dos Centros de Testagem e Aconselhamento (CTA) no Brasil em 1988, oferecendo, entre outras atividades, o aconselhamento e a realização de testes sorológicos anti-HIV e para outras Infecções Sexualmente Transmissíveis (IST). Tais centros permitem conhecer e avaliar de maneira precoce o perfil epidemiológico dos infectados, auxiliando nas respostas a operações políticas e sociais no controle e tratamento da doença, além de garantir o vínculo, acolhimento, privacidade do usuário e confidencialidade dos dados (7). Mesmo assim, alguns estudos reforçam que, nos CTA, o aconselhamento pré e pós testagem é ainda deficiente, com desenvolvimento insuficiente de algumas ações ${ }^{(8)}$.

No campo da assistência, importante feito tem sido observado no que tange ao tempo de vida dos portadores, que apresentou elevação considerável, especialmente pela adesão aos antirretrovirais, muito embora o número de indivíduos com dificuldades de aceitação ainda permaneça elevado ${ }^{(9)}$. Para dirimir essa realidade, é necessário criar uma relação de confiança entre profissional de saúde e usuário num ambiente livre de estigma e discriminação, de forma a tornar o indivíduo ativo no cuidado de si e referência para o controle da doença ${ }^{(5,8)}$.

Destarte, torna-se necessário entender mais sobre a temática, principalmente por meio de estudos que delineiem o perfil epidemiológico e clínico de usuários dos centros de referências para HIV/Aids, especialmente daqueles localizados em cidades de pequeno e médio portes, onde ainda permanecem ignorados ${ }^{(1)}$. Para tanto, esta pesquisa teve por objetivo conhecer o perfil epidemiológico dos usuários atendidos no serviço de referência para IST/HIV/Aids do município de Petrolina-PE. 


\section{Método}

Trata-se de estudo descritivo com abordagem quantitativa realizado no Serviço Ambulatorial Especializado - (SAE) em Petrolina, Pernambuco. O SAE está localizado no 'Espaço Vida', local no qual funciona também o Centro de Testagem e Aconselhamento - (CTA), que realiza testes rápidos anti-HIV, $\mathrm{HBsAg}$, anti-HCV e sífilis, além de disponibilizar preservativos e materiais educativos, cuja média diária é de 200 atendimentos. O SAE realiza acompanhamento terapêutico, que inclui atendimento médico especializado, consulta de enfermagem e apoio psicológico aos usuários com diagnóstico de DST/HIV/Aids, além de controlar a distribuição de antirretrovirais e realizar exames de monitoramento, contando com equipe multiprofissional que atende à clientela da cidade e de municípios circunvizinhos, sendo um serviço de referência para a região.

Os dados foram coletados por meio das fichas de notificação e investigação para HIV/Aids da clientela atendida no serviço. A amostra foi constituída por todas as fichas dos usuários cadastrados no SAE no período de 2010 a 2014, independentemente da data de notificação, cor, raça, profissão e nível de escolaridade, totalizando 331 notificações.

A coleta de dados somente teve início após aprovação pelo Comitê de Ética da Universidade Federal do Vale do São
Francisco (UNIVASF) (protocolo $\mathrm{n}^{\mathrm{o}}$ 0008/060215 - CEDEP). Foram respeitadas as Diretrizes e Normas Regulamentadoras de pesquisa envolvendo seres humanos definidas pela Resolução 466/12, garantindo o sigilo dos dados dos participantes.

Inicialmente, aplicou-se a estatística descritiva às variáveis sociodemográficas e clínicas por meio da distribuição de frequência absoluta e relativa, adotando-se intervalos de confiança de $95 \%$ para as proporções e assumindo uma distribuição binomial.

A variável 'idade' foi analisada por meio de medidas de tendência central e dispersão, como média e desvio padrão, assim como o intervalo de confiança de $95 \%$ para média. Após a verificação da normalidade da distribuição normal pelo teste Shapiro Wilk, verificou-se a existência de diferença na média de idade para dois grupos independentes segundo as variáveis 'sexo' (masculino ou feminino), 'zona de residência' (urbana ou rural), 'município de residência' (Petrolina ou outros municípios), 'raça/cor' (branca ou não branca) e 'provável modo de transmissão' (sexual ou outros). Em seguida, testou-se a significância estatística por meio do teste $t$ de Student, sendo considerados significantes valores de $\mathrm{p}<0,05$. Posteriormente, a idade foi categorizada segundo faixa etária.

Para avaliação da tendência de ocorrência dos casos de Aids registrados no 
serviço, foi realizada regressão linear, com correção de erros robustos, tendo como variável dependente o 'número de casos notificados' e como variável preditora, o 'ano de diagnóstico'. Para o teste, considerou-se tendência de crescimento, a positividade do coeficiente e o valor de $\mathrm{p}<0,05$. Foi avaliada a tendência de evolução dos casos nas faixas etárias e sexo.

Os dados foram tratados por meio do software estatístico Stata 12.0 e as tabelas e gráfico, editados no Programa Microsoft Office Excel 2013. Quanto ao critério de diagnóstico Rio de Janeiro-Caracas, considerando que os registros possuíam em sua maioria, mais de um critério, optou-se por construir variáveis binárias, agregando a de análise e as demais. Os sintomas foram analisados de forma separada: diarreia, astenia ou caquexia, tosse persistente, febre maior ou igual a $38^{\circ} \mathrm{C}$, tuberculose extra-pulmonar, anemia ou linfadenopatia, Sarcoma ou disfunção do SNC, candidose ou Herpes Zoster ou dermatite. Os pacientes que possuíam pelo menos um desses diagnósticos foram separados dos demais e analisados individualmente.

\section{Resultados}

Analisaram-se 331 prontuários de indivíduos infectados pelo HIV atendidos no serviço especializado municipal de 2009 a 2014. Os dados encontram-se dispostos nos gráficos 1 e 2 e tabelas 1,2 e 3 . Analisando-se a tendência da evolução do número de casos de Aids para o período, observou-se tendência de crescimento ( $p<0,0001)$ (Gráfico 1). Essa tendência também foi observada para as faixas etárias e para o sexo $(\mathrm{p}<0,0001)$ (Gráfico 2).

Gráfico 1- Casos de Aids de acordo com a faixa etária e o ano de notificação.

\begin{tabular}{|c|c|c|c|c|c|c|c|}
\hline \multirow{5}{*}{ 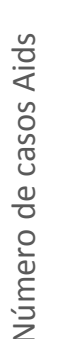 } & \multicolumn{7}{|l|}{30} \\
\hline & \multicolumn{7}{|l|}{20} \\
\hline & \multicolumn{7}{|l|}{15} \\
\hline & \multicolumn{7}{|l|}{5} \\
\hline & 0 & $20 \overline{0}$ & 2010 & 301 & 2012 & 2013 & 2014 \\
\hline & & 1 & 7 & 2 & 0 & 0 & 3 \\
\hline & & 0 & 1 & 0 & 3 & 1 & 5 \\
\hline & & 8 & 7 & 7 & 9 & 9 & 20 \\
\hline & & 9 & 10 & 17 & 24 & 21 & 21 \\
\hline & & 10 & 11 & 7 & 11 & 14 & 15 \\
\hline & & 3 & 6 & 8 & 4 & 11 & 6 \\
\hline & mais & 1 & 3 & 5 & 1 & 4 & 2 \\
\hline
\end{tabular}


Em 2014, houve o maior número de notificações, 72 casos, sendo $29 \%$ do total desde 2009. De acordo com os critérios de notificação da doença, modificados em 2014, a notificação de infecção por HIV passou a ser compulsória, revelando que $92,7 \%$ dos 331 pacientes tinham Aids e 7,3\%, HIV.

Gráfico 2- Casos de Aids de acordo com o gênero e ano de notificação

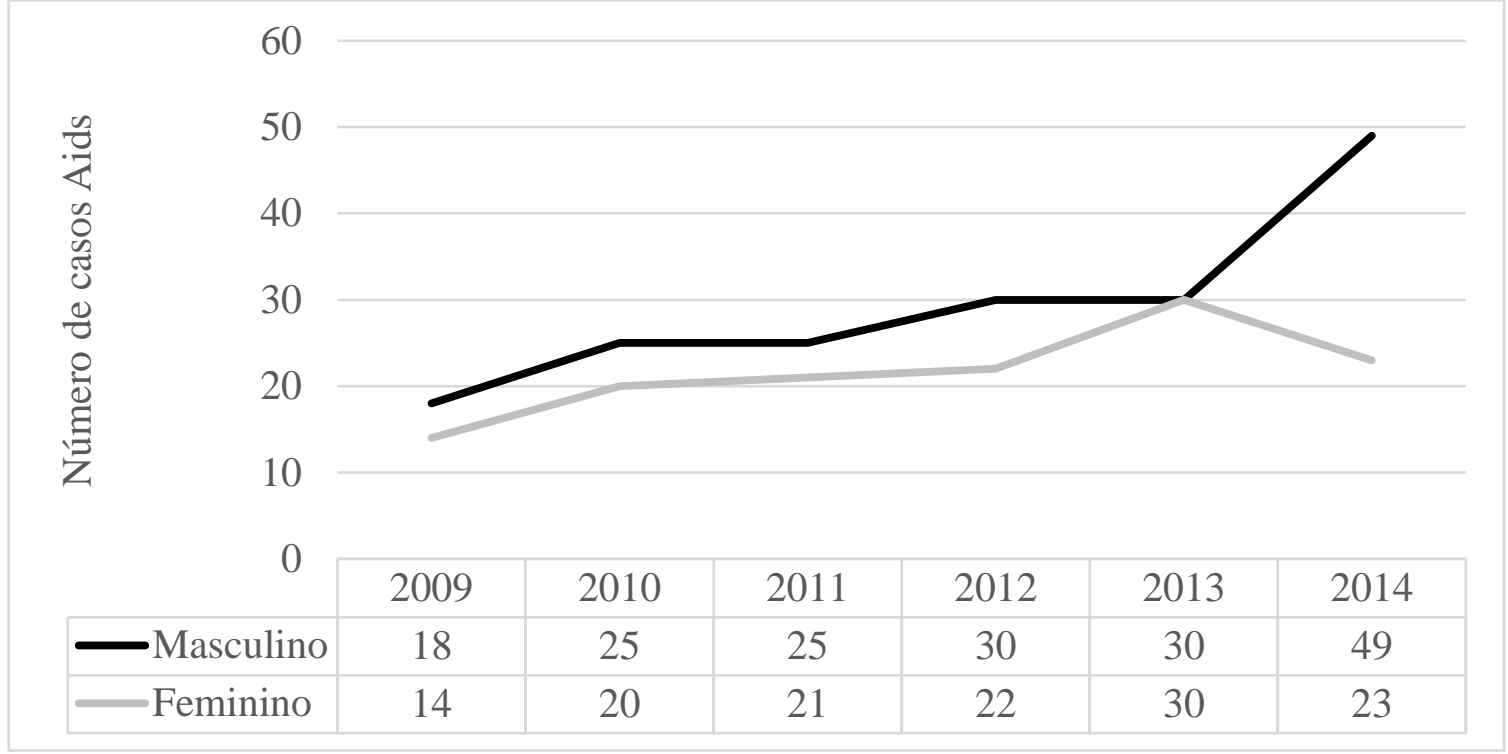

Quanto às características

sociodemográficas, houve predominância do sexo masculino, de população jovem adulta e raça parda. A maioria possuía apenas ensino fundamental (Tabela 1). Observou-se também que não houve diferença significativa entre as médias de idades dos homens e das mulheres $(\mathrm{p}=0,2731)$, de idade entre pessoas residentes em Petrolina ou em outros municípios, $(\mathrm{p}=0,9031)$ ou entre pessoas segundo a zona de residência $(\mathrm{p}=0,6934)$.

Tabela 1 - Distribuição de frequência das características sociodemográficas da clientela do CTA Petrolina.

\begin{tabular}{llccc}
\hline Variáveis Sociodemográficas & $\mathbf{n}$ & $\mathbf{\%}$ & \multicolumn{2}{c}{ IC 95\%* } \\
\hline Sexo $(\mathbf{n}=\mathbf{3 3 1})$ & 138 & 41,7 & 36,4 & 47,0 \\
Feminino & 193 & 58,3 & 53,0 & 63,6 \\
Masculino & & & & \\
Faixa etária em anos $(\mathbf{n}=\mathbf{3 3 1})$ & 13 & 3,9 & 1,8 & 6,0 \\
Até 13 & 12 & 3,6 & 1,6 & 5,6 \\
14 a 19 & & & &
\end{tabular}




$\begin{array}{lllll}20 \text { a } 29 & 71 & 21,5 & 17,0 & 25,9 \\ 30 \text { a } 39 & 106 & 32,0 & 27,0 & 37,1 \\ 40 \text { a } 49 & 72 & 21,8 & 17,3 & 26,2 \\ 50 \text { a } 59 & 40 & 12,1 & 8,6 & 15,6 \\ 60 \text { ou mais } & 17 & 5,1 & 2,7 & 7,5\end{array}$

Raça-cor $(n=329)$

Branca

$\begin{array}{llll}6 & 1,8 & 0,4 & 3,3\end{array}$

Preta

0,6

$-0,2$

Parda

97,6

95,9

99,2

Zona de residência $(n=330)$

Rural

35

Urbana

10,6

7,3

13,9

Escolaridade $(\mathbf{n}=\mathbf{3 3 1})$

Ensino Fundamental

Ensino Médio

90

27,2

22,4

32,0

Ensino Superior

28

8,5

5,4

11,5

Não Alfabetizado

12

3,6

1,6

5,6

Desconhecido

45

13,6

9,9

17,3

Município de residência $(n=331)$

Outros Municípios

60

$18,1 \quad 14,0$

22,3

Petrolina

*Intervalo de Confiança 95\% para proporção, assumindo distribuição binomial

O provável modo de transmissão teve maior concentração na via sexual. $O$ contato considerado na transmissão sexual foi, em sua maioria, homens. Observou-se uma letalidade de $2,1 \%$. A principal evidência laboratorial para o diagnóstico foi a triagem Anti-HIV ou a testagem rápida (Tabela 2). A média de idade para transmissão sexual foi superior às outras formas de transmissão, sendo a média de idade para transmissão sexual de 38,3 anos (p <0,0001; DP 12,3; IC95\% 36,8 - 39,7). 
Tabela 2 - Distribuição das características clínicas dos pacientes atendidos no CTA do município de Petrolina.

\begin{tabular}{|c|c|c|c|}
\hline Variáveis clínicas & $\mathbf{n}$ & $\%$ & IC95\%* \\
\hline \multicolumn{4}{|l|}{ Contato sexual Transmissão $(n=282)$} \\
\hline Relação com mulher & 102 & 36,2 & $30,5-41,8$ \\
\hline Relação com homens & 177 & 62,8 & $57,1-68,4$ \\
\hline Relação com ambos & 3 & 1,1 & $-0,1-2,3$ \\
\hline \multicolumn{4}{|l|}{ Provável modo de transmissão $(\mathbf{n}=297)$} \\
\hline Sexual & 280 & 94,3 & $91,6-96,9$ \\
\hline Vertical & 14 & 4,7 & $2,3-7,1$ \\
\hline Sanguínea & 3 & 1,0 & $-0,1$ a 2,1 \\
\hline \multicolumn{4}{|l|}{ Caso Aids $(n=331)$} \\
\hline Não & 24 & 7,3 & $4,4-10,1$ \\
\hline Sim & 307 & 92,8 & $89,9-95,6$ \\
\hline \multicolumn{4}{|l|}{ Evolução do Caso $(n=331)$} \\
\hline Vivo & 304 & 91,8 & $88,9-94,8$ \\
\hline Óbito & 7 & 2,1 & $0,6-3,7$ \\
\hline Mãe falecida por Aids & 2 & 0,6 & $0,2-1,4$ \\
\hline Desconhecido & 18 & 5,4 & $3,0-7,9$ \\
\hline \multicolumn{4}{|l|}{ Evidência laboratorial (n = 321) } \\
\hline Teste de triagem anti-HIV reagente & 115 & 35,8 & $30,6-41,1$ \\
\hline Teste rápido reagente & 102 & 31,8 & $26,6-36,9$ \\
\hline $\begin{array}{l}\text { Teste de triagem anti-HIV e teste rápido } \\
\text { reagente }\end{array}$ & 90 & 28,0 & $23,1-33,0$ \\
\hline Teste confirmatório & 11 & 3,4 & $1,4-5,4$ \\
\hline Teste de detecção de ácido nucléico & 2 & 0,6 & $-0,2-1,5$ \\
\hline $\begin{array}{l}\text { Teste de detecção de ácido nucléico e teste de } \\
\text { triagem anti-HIV }\end{array}$ & 1 & 0,3 & $-0,3-0,9$ \\
\hline \multicolumn{4}{|l|}{ Gestante $(n=138)$} \\
\hline Não & 133 & 96,4 & $93,2-99,5$ \\
\hline Sim & 5 & 3,6 & $0,5-6,8$ \\
\hline
\end{tabular}

Houve quatorze notificações de pessoas menores de treze anos, todas elas infectadas por transmissão vertical. Nesse recorte, sete eram do sexo masculino e duas tiveram suas mães falecidas por Aids.

Quanto à frequência dos critérios utilizados para definição de caso, o critério CDC adaptado teve seus achados prevalentes em $53,5 \%$ dos casos no momento do diagnóstico. o critério Rio de Janeiro-Caracas teve seus achados prevalentes em $42,6 \%$ dos casos no momento do diagnóstico os pacientes HIV assintomáticos totalizaram 7,3\% dos casos, com diagnóstico realizado por meio de evidência laboratorial.

No que diz respeito aos achados clínicos do critério Rio de Janeiro-Caracas, houve prevalência dos sintomas diarreia, 
astenia ou caquexia (93\%); anemia ou linfadenopatia $(43,4 \%)$; febre maior ou igual a $38^{\circ} \mathrm{C}(42 \%) ;$ e tosse persistente $(32,2 \%)$ (Tabela 3). Já o critério CDC adaptado teve como maior achado a contagem de linfócitos
T CD4+ menor do que $350 \mathrm{cel} / \mathrm{mm}^{3}$ em $98,9 \%$ dos casos, a neurotoxoplasmose $(1,7 \%)$, citomegalovirose $\quad(1,1 \%), \quad$ isosporidiose $(0,6 \%)$ e criptosporidiose intestinal crônica $(0,6 \%)$.

Tabela 3 - Critério Rio de Janeiro-Caracas de maior prevalência na clientela do CTA Petrolina.

\begin{tabular}{lrrr}
\hline & n & \% & IC95\%* \\
\hline Outros diagnósticos & 10 & 7,0 & $2,8-11,2$ \\
Diarreia, Astenia ou caquexia & 133 & 93,0 & $88,8-97,2$ \\
& & & \\
Outros diagnósticos & 97 & 67,8 & $60,1-75,6$ \\
Tosse persistente & 46 & 32,2 & $24,4-39,9$ \\
& & & \\
Outros diagnósticos & 83 & 58,0 & $49,8-66,2$ \\
Febre maior ou igual a 38'C & 60 & 42,0 & $33,8-50,1$ \\
& & & \\
Outros diagnósticos & 125 & 87,4 & $81,9-92,9$ \\
Tuberculose extrapulmonar & 18 & 12,6 & $7,1-18,1$ \\
& & & \\
Outros diagnósticos & 81 & 56,6 & $48,4-64,9$ \\
Anemia ou linfadenopatia & 62 & 43,4 & $35,1-51,6$ \\
& & & \\
Outros diagnósticos & 141 & 98,6 & $96,7-100,5$ \\
Sarcoma ou Disfunção SNC & 2 & 1,4 & $-0,5-3,3$ \\
Outros diagnósticos & & & \\
Candidose ou Herpes Zoster ou dermatite & 108 & 75,5 & $68,4-82,7$ \\
\hline
\end{tabular}

*Intervalo de Confiança 95\% para proporção assumindo distribuição binomial

\section{Discussão}

O grande aumento no número de notificações no ano 2014 no Município era esperado, assim como nos demais municípios da federação. Cabe destacar que esse episódio se deve ao fato que, até 2014, os casos de infecção pelo vírus HIV somente eram notificados quando do surgimento da Aids. Porém, a Portaria no 1.271, de 6.6.2014, incluiu a infecção por HIV na Lista Nacional de Notificação Compulsória de Doenças, Agravos e Eventos de Saúde Pública. Antes, a obrigatoriedade era restrita aos casos de infecção em gestante, parturiente, puérpera e criança exposta ao risco de transmissão vertical do vírus. Diante desse novo contexto, é provável que o número de notificações 
aumente vertiginosamente, até que haja tendência de estabilização.

A modificação no perfil dos acometidos pela infecção do vírus HIV trouxe, consequentemente, mudanças na população infectada, que antes se concentrava em grandes centros urbanos e, atualmente, está presente também em regiões de menor porte, como é o caso do município de Petrolina, situada no interior do estado de Pernambuco, assim como nas cidades circunvizinhas que utilizam os serviços de referência.

Tal fenômeno de expansão e interiorização da epidemia despertou a iniciativa dos serviços de vigilância em saúde para investigação e detecção de novos casos do vírus HIV. Estudo realizado no estado do Rio Grande do Norte revela o aumento da taxa de crescimento nas microrregiões, da mesma forma que pesquisa realizada no interior de Minas Gerais. Apesar da expansão e disseminação da infecção nas localidades de menor porte, ainda não é possível realizar equiparação da epidemia, que permanece com maiores concentrações nas capitais e grandes cidades ${ }^{(1,10-11)}$.

No que tange à interiorização da epidemia de HIV/Aids, a elevação do número de casos pode ser explicada por fatores sociais e culturais tias como pobreza, baixa escolaridade e renda e deficiência dos serviços de saúde pelo isolamento geográfico dos grandes centros urbanos, o que parece distanciar sua população de maiores informações acerca da Aids e de práticas de prevenção. Talk realidade revela que a preocupação com a doença ainda não chegou às pequenas cidades ${ }^{(12)}$.

No quesito zona de moradia e acessibilidade ao serviço, observou-se que a maioria dos usuários era residente da zona urbana do município, o que pode ter facilitado o acesso ao diagnóstico, sendo este desfavorável às populações da zona rural pela distância geográfica dos centros especializados, que se concentram nas áreas urbanas. Muitos também provinham de localidades vizinhas, atendidos pelo serviço por motivos distintos, dentre eles a falta de suporte em sua localidade de origem ou o medo do vazamento de informações sobre o diagnóstico e tratamento da doença. Sabe-se que a estigmatização dessa doença ainda é um fato, bem como a permanência do preconceito, gerando dificuldades, inclusive, na adesão às terapias prescritas ${ }^{(2,6)}$. Sendo assim, enquanto tais problemas persistirem, a abordagem às populações, especialmente àquelas acometidas, será tolhida.

Este estudo corrobora os achados descritos pela literatura ${ }^{(2,5)}$, ressaltando que, apesar da disseminação da infecção no país, o sexo masculino ainda permanece com maior prevalência dos casos, remetendo ao estilo de vida sem muitas precauções, principalmente em relação à saúde, ainda negligenciada pelo sexo masculino. 
No entanto, não se pode negligenciar o aumento vertiginoso de mulheres infectadas, o que traduz a vulnerabilidade feminina para o adoecimento em razão das desigualdades de gênero. É necessário, então, atuar para desmistificar tabus existentes acerca do contágio, minimização do preconceito e dos medos que interferem na vida delas. Os profissionais de saúde devem orientá-las sobre a prevenção da transmissão do vírus para possíveis parceiros (as), como também acerca da importância do tratamento, na tentativa de que mantenham uma vida normal $(5,13)$.

No tocante à faixa etária, outros estudos também demonstraram perfil etário semelhante, revelando que a infecção tem se concentrado em população jovem adulta, o que se justifica por ser este um grupo que possui vida sexual ativa. Por essa razão, a possibilidade de educação em saúde para mudanças nas práticas sexuais tem suas dificuldades potencializadas, inclusive entre portadores de HIV/Aids, pois as atitudes já estão cristalizadas, tornando necessário investir em ações que viabilizem a interação entre essa população e profissionais de saúde no intuito de dialogar sobre a temática ${ }^{(8,14-15)}$.

No que concerne à escolaridade, esse indicador é utilizado para avaliar a situação socioeconômica da população. Assim, os dados revelaram que a clientela estudada, em sua maioria, possuía baixa escolaridade, corroborando outra pesquisa ${ }^{(14)}$, que revelou mudança no padrão das possíveis causas da transmissão do vírus ao nível de formação escolar, inferindo provável ligação entre pauperização e contágio. A baixa escolaridade influencia, inclusive, a adesão à terapia antirretroviral, que é prejudicada em indivíduos com menor escolarização ${ }^{(9)}$.

A tríade população mais pobre-baixa escolaridade-disseminação do vírus configura um grupo em maior vulnerabilidade para o HIV em razão do menor acesso à informação, medidas preventivas e possibilidade de se beneficiar com novos conhecimentos (14). Atrelado a esses fatos, o cenário em que foi desenvolvida a pesquisa ainda possui elevadas taxas de analfabetismo e baixa escolaridade, que, aliadas a outros fatores descritos, parecem potencializar a vulnerabilidade a várias comorbidades, inclusive a infecção pelo HIV.

O número de infectados com ensino superior completo neste estudo mostrou-se inexpressivo $(6,6 \%)$, ratificando o perfil nacional. Os índices também revelaram baixo nível de escolaridade entre as mulheres. Sabese que a mulher com menor grau de instrução também pode ser mais vulnerável à exposição, especialmente porque a feminização da Aids envolve fatores biológicos, dependência financeira, falta de autonomia, com baixo poder do exercício de sua sexualidade, e a relação que a mulher faz com o uso de preservativo, vinculando-o 
apenas às questões de contracepção e não como forma de prevenção das IST ${ }^{(16)}$.

Os índices encontrados por esta pesquisa têm como característica predominante a transmissão sexual, especialmente no que tange às relações entre homem e mulher, uma vez que todos os casos de mulheres infectadas ocorreram por relação sexual apenas com homens, indicando heterossexualização da infecção. Esse dado reafirma e reflete o que descreve a literatura acerca da cultura latina, que perpassa comportamentos nos quais os homens não devem se opor a manter relações sexuais com outras mulheres, na maioria das vezes desprotegidas, mesmo que tenham relação conjugal estável, promovendo a promiscuidade e transmissão de doenças por via sexual (15). Esse fenômeno de heterossexualização da infecção pelo vírus HIV também é um fator que tem contribuído para a feminização da epidemia de Aids no cenário nacional, como revelado por estudos sobre o tema ${ }^{(2,11,16)}$.

Em paralelo, verificou-se que práticas homossexuais foram relatadas em casos notificados, todos praticados entre homens, como encontrado em estudo sobre a interiorização do HIV no Brasil (11), que mostrou comportamento homossexual em $22,2 \%$ dos homens e $0,6 \%$ nas mulheres com HIV/Aids. Tais achados demonstram que a síndrome não mais pode ser atrelada simplesmente ao homossexualismo, como outrora, mas, sim, aos diferentes grupos populacionais, que se tornam vulneráveis aos fatores de risco para contrair o vírus devido às atitudes sexuais desprotegidas, em sua maioria.

Em relação ao número de casos notificados em crianças menores de treze anos, segundo dados do DATASUS, no período de 2010 a 2014, o estado de Pernambuco notificou 99 casos de transmissão vertical, concentrando $14,1 \%$ deles no Município em estudo. Diante do cenário nacional, que vem viabilizando medidas para detecção de novos casos de maneira rápida e eficaz, tais números podem estar em desacordo, uma vez que em 2013 foram identificadas 7.219 gestantes infectadas pelo HIV, sendo 19,2\% na região Nordeste. Levando-se em consideração a probabilidade de pré-natal inadequado e possível infecção durante o parto, esses números podem ter sofrido subnotificações.

Vale ressaltar que o diagnóstico de Aids em crianças é cercado de dificuldades, pois envolve o círculo familiar, além do medo e de diferenças biológicas e reacionais no curso da adesão ao tratamento. Estudo que descreveu como as famílias e pessoas com vínculo com crianças com HIV reagiram ao diagnóstico e como se deu o curso terapêutico observou que, na maioria dos casos, o silêncio foi estabelecido como medida de proteção para garantir a integridade física, emocional e social do grupo familiar e da criança ${ }^{(17)}$. 
Dentre os achados clínicos de infecções oportunistas, encontram-se $\mathrm{o}$ critério Rio de Janeiro-Caracas e o CDC Adaptado, que determinam aspectos de suspeita clínica da infecção. O primeiro é utilizado para verificar a presença de sintomatologia característica da síndrome a partir de uma escala com sinais e sintomas elencados, que, se somados dez pontos associados a dois testes de triagem reagentes ou um teste confirmatório, caracterizam e diagnosticam a instalação da infecção da Aids. Neste estudo, tal critério teve como maior prevalência a astenia e caquexia, além de diarreia crônica e febre por mais de um mês, que são os mais comuns entre os acometidos pelos vírus. Já o critério CDC Adaptado, evidencia a imunodeficiência no indivíduo a partir da contagem de Linfócitos CD4+ menor que 350 cél $/ \mathrm{mm}^{3}$ ou de qualquer um dos sintomas descritos, tendo sido prevalentes para neurotoxoplasmose após a contagem.

Diante das mudanças no perfil epidemiológico da Aids no decorrer dos anos, a mortalidade por essa síndrome no Brasil também foi afetada. Na população adulta, observou-se redução de $11,1 \%$ na mortalidade pela doença no país, seja pela adesão aos antirretrovirais ou por outros fatores, embora a contaminação ainda se eleve em muitos lugares, inclusive na região deste estudo ${ }^{(18)}$. A redução da mortalidade também pode ser um fator que leve à despreocupação com a contaminação pela doença, uma vez que a letalidade tem sido cada vez menor em relação aos anos anteriores, necessitando de diálogo com a sociedade para desmitificar a questão.

Finalmente, destaca-se a importância das ações de cunho educativo e preventivo na saúde pública para tal enfermidade, por meio do foco em medidas que ressaltem a importância da prática sexual protegida pelo uso de preservativo para todas as populações jovens, adultos e idosos -, já que nenhuma delas está imune à infecção. Uma vez que o conhecimento da população sobre as formas de transmissão ainda é insatisfatório, justificado pelas práticas vulneráveis que ainda adotam, é necessário investir em políticas públicas eficazes que denotem a real situação e chamem à atenção para que a vulnerabilidade seja minimizada, conscientizando sobre o sexo seguro e evitando, assim, novos casos de contaminação pelo HIV.

\section{Considerações finais}

O perfil epidemiológico daqueles que fizeram parte do estudo equipara-se aos dados encontrados no território nacional, que revela, ainda, o predomínio do sexo masculino em idade economicamente ativa e baixo nível de escolaridade. No entanto, acompanhando o cenário brasileiro, preocupa o resultado dos índices referentes à infecção no sexo 
feminino, já que se percebe aumento crescente nas notificações também na região e localidades circunvizinhas atendidas pelo serviço estudado.

É importante ressaltar que as políticas de prevenção e diagnóstico precoce devem incluir atividades de educação em saúde diferenciadas e voltadas para todos os públicos. É comum observar resistência ao uso do preservativo, como também dificuldade de entendimento acerca das informações sobre tais questões. Sendo assim, urge a necessidade de avaliar as políticas públicas adotadas, bem como suas ações programáticas, melhorando a qualidade do atendimento ofertado no serviço.

A cultura brasileira também conjectura para a vulnerabilidade dos dois sexos, colocando à mulher em situação de desvantagem acerca da força e virilidade masculina, culturalmente reforçada e perpassada, contribuindo para a propagação de várias doenças transmitidas por via sexual, incluindo o HIV/Aids.

Espera-se que os dados revelados por esta pesquisa contribuam para 0 direcionamento de ações de prevenção, promoção e controle do HIV/Aids, como também para a adoção de políticas adequadas para interrupção da transmissão, realização de diagnóstico precoce e implementação do tratamento. Desse modo, reconhecer as características epidemiológicas locais pode proporcionar a formulação de ações de saúde específicas, embora não seja uma doença que permita generalizações. 


\section{Referências}

1. Bezerra JS, Barbosa CR. Relações de Gênero e a Feminização do HIV/Aids. Anais do Congresso Internacional da Faculdades EST. São Leopoldo: EST, v. 2, 2014.

2. Brasil. Boletim Epidemiológico - Aids e DST - ano V número I. Brasília: Ministério da Saúde, 2016. Disponível em: http://www.aids.gov.br/sites/default/files/anex os/publicacao/2016/59291/boletim_2016_1_p df_16375.pdf

3. Brito FG, Madi RR, Rezende MIRC, Melo CM. Perfil Epidemiológico de Portadores do Vírus da Imunodeficiência Humana e Síndrome da Imunodeficiência Adquirida no Estado de Sergipe, 2007-2012. Interfaces Científicas - Saúde e Ambiente. Aracaju, Fev 2014.

4. Campos CGAP,Estima SL, Santos VS, Lazzarotto AR. A Vulnerabilidade ao HIV em adolescentes: Estudo retrospectivo em um Centro de Testagem e Aconselhamento. Revista Mineira de Enfermagem. 2014 abr/jun; 18(2): 310-314.

5. Faqueti A, Rodriguez AMM, Woerner CB, Antonio GD. Perfil Epidemiológico de Mortalidade por Aids na População Adulta do Brasil de 2001 a 2010. Rev. Saúde Públ. Santa Cat., Florianópolis, v. 7, n. 2, p. 29-39, maio/ago. 2014.

6. Filho Oliveira de JS, Silva PE, Freitas FFQ, Soares JP, Costa MAG, Silva e ACO. Qualidade de Vida de Pessoas Vivendo com HIV/Aids. Revista Baiana de Enfermagem, Salvador, v. 28, n. 1, p. 61-68, jan./abr. 2014.

7. Furtado FM, Santos J, Stedile L, Araújo E, Saldanha A. Interiorização da Aids: Representações sociais de residentes de cidades rurais. Investigação Qualitativa em Saúde. Porto-Pt, Vol 1. P. 64-68.

8. Oliveira ADF, Vieira MCA, Silva SPC, Mistura C, Jacobi CS, Lira MOSC. Repercussões do HIV no cotidiano de mulheres vivendo com Aids. J. res.: fundam. care. online 2015. jan./mar. 7(1):1975-1986.

9. Paula de CC, Padoin SMM, Langendorf TF, Mutti CF, Hoffmann IC, Valadão MC. Acompanhamento Ambulatorial de Crianças que tem HIV/Aids: Cuidado Centrado na Criança e na Família. Ciênc. Cuid. Saúde. 2012 Jan/Mar.
10. Pereira BS, Costa MCO, Amaral MTR, Costa da HS, Silva da CAL, Sampaio VS. Fatores associados à infecção pelo HIV/AIDS entre adolescentes e adultos jovens matriculados em Centro de Testagem e Aconselhamento no Estado da Bahia, Brasil. Ciência \& Saúde Coletiva, 19(3):747-758, 2014.

11. Santos ÉI., Silva AL., Santana PPC., Teixeira PA. Evidências científicas brasileiras sobre adesão à terapia antirretroviral por pessoas que vivem com HIV/AIDS. Revista Eletrônica Gestão \& Saúde, Vol.07, Nº. 01, Ano 2016 p. 454-70.

12. Schaurich D, Freitas, HMB. O referencial de vulnerabilidade ao HIV/AIDS aplicado às famílias: um exercício reflexivo. Rev. Esc. Enferm. USP. 45(4):989-95, 2011.

13. Schuelter-Trevisol F, Pucci P, Justino AZ, Pucci N, Silva da ACB. Perfil epidemiológico dos pacientes com HIV atendidos no sul do Estado de Santa Catarina, Brasil, em 2010. Epidemiol. Serv. Saúde, Brasília, 22 (1):8794, jan-mar 2013.

14. Silva AP, Machado PRF, Martins ERC, Costa CMA, Alves RN, Ramos RCA. Conhecimento e Percepção de Vulnerabilidades para o HIV/Aids entre os acadêmicos de uma universidade privada. Revista de Enfermagem UERJ. Rio de Janeiro, 2013 dez; 21(esp.1): 618-23.

15. Silva ITS, Silva DC, Salvetti GM, Torres GV, Silva RAR, Souza NL. Perfil dos Casos de Síndrome da Imunodeficiência Adquirida em Um Estado do Nordeste do Brasil. Rev Enferm UFSM 2014 Out/Dez;4

16. Souza de CC, Mata da LRF, Azevedo C, GOMES CRG, Cruz GEC Toffano SEM. Interiorização do HIV/Aids no Brasil: Um Estudo Epidemiológico. Revista Brasileira de Ciências da Saúde, ano 11, n 35, jan/mar. 2013.

17. Taquette SR., Rodrigues AO., Bortolotti LR. Percepção de pacientes com AIDS diagnosticada na adolescência sobre $o$ aconselhamento pré e pós-teste HIV realizado. Ciência \& Saúde Coletiva, 22(1):23-30, 2017

18. UNAIDS. GLOBAL AIDS UPDATE 2016. Disponível em: http://www.unaids.org/sites/default/files/medi a_asset/global-AIDS-update-2016_en.pdf. 
Revista Eletrônica Gestão \& Saúde ISSN: 1982-4785

Santos NTN, Silva SPC, Fernandes FECV et al

Perfil epidemiológico de casos HIV/Aids...

\section{Participação dos autores:}

SANTOS, NTN trabalhou na concepção teórica, coleta de dados, análise estatística e elaboração e redação final do texto;

SILVA, SPC trabalhou na concepção teórica, Recebido: 17.04.2017 elaboração, análise estatística e redação final do texto; FERNANDES, FECV trabalhou na análise estatística; Revisado: 07.03.2018

Santana, LD trabalhou redação final do texto; Aprovado:18.04.2018

SILVA, TIM trabalhou na redação final do texto. 